\title{
Mobile Genetic Elements Harboring Antibiotic Resistance Determinants in Acinetobacter baumannii Isolates From Bolivia
}

\begin{abstract}
Mónica Cerezales ${ }^{1 *}$, Kyriaki Xanthopoulou ${ }^{2,3+}$, Julia Wille ${ }^{2,3}$, Oleg Krut ${ }^{4}$, Harald Seifert ${ }^{2,3}$, Lucía Gallego ${ }^{1}$ and Paul G. Higgins ${ }^{2,3}$

${ }^{1}$ Faculty of Medicine and Nursing, Department of Immunology, Microbiology, and Parasitology, University of the Basque Country UPVIEHU, Leioa, Spain, ${ }^{2}$ Institute for Medical Microbiology, Immunology and Hygiene, University of Cologne, Cologne, Germany, ${ }^{3}$ German Centre for Infection Research (DZIF), Partner Site Bonn-Cologne, Cologne, Germany,

${ }^{4}$ Paul-Ehrlich-Institute, Federal Institute for Vaccines and Biomedicine, Langen, Germany
\end{abstract}

\section{OPEN ACCESS}

Edited by:

Benjamin Andrew Evans, University of East Anglia,

United Kingdom

Reviewed by:

Nabil Karah,

Umeå University, Sweden Andres Felipe Opazo-Capurro,

University of Concepcion, Chile

*Correspondence:

Mónica Cerezales

mcerezales001@ikasle.ehu.eus;

monicacerezalesg@gmail.com

†These authors have contributed equally to this work

Specialty section: This article was submitted to Antimicrobials, Resistance and Chemotherapy,

a section of the journal Frontiers in Microbiology

Received: 15 January 2020 Accepted: 17 April 2020 Published: 13 May 2020

Citation:

Cerezales M, Xanthopoulou K, Wille J, Krut O, Seifert H, Gallego L and Higgins PG (2020) Mobile Genetic Elements Harboring Antibiotic Resistance Determinants in Acinetobacter baumannii Isolates From Bolivia.

Front. Microbiol. 11:919 doi: 10.3389/fmicb.2020.00919
Using a combination of short- and long-read DNA sequencing, we have investigated the location of antibiotic resistance genes and characterized mobile genetic elements (MGEs) in three clinical multi-drug resistant Acinetobacter baumannii. The isolates, collected in Bolivia, clustered separately with three different international clonal lineages. We found a diverse array of transposons, plasmids and resistance islands related to different insertion sequence (IS) elements, which were located in both the chromosome and in plasmids, which conferred resistance to multiple antimicrobials, including carbapenems. Carbapenem resistance might be caused by a Tn2008 carrying the blaOXA-23 gene. Some plasmids were shared between the isolates. Larger plasmids were less conserved than smaller ones and they shared some homologous regions, while others were more diverse, suggesting that these big plasmids are more plastic than the smaller ones. The genetic basis of antimicrobial resistance in Bolivia has not been deeply studied until now, and the mobilome of these A. baumannii isolates, combined with their multi-drug resistant phenotype, mirror the transfer and prevalence of MGEs contributing to the spread of antibiotic resistance worldwide and require special attention. These findings could be useful to understand the antimicrobial resistance genetics of $A$. baumannii in Bolivia and the difficulty in tackling these infections.

Keywords: A. baumannii, plasmids, mobile genetic elements, antimicrobial resistance, carbapenemase

\section{INTRODUCTION}

Acinetobacter baumannii is a non-fermenting Gram-negative bacilli and it is the second most common species after Pseudomonas aeruginosa in this group causing bacterial infections (Gonzalez-Villoria and Valverde-Garduno, 2016). While A. baumannii has been isolated from the wider environment such as water, soil, and animals, most studied isolates come from clinical samples, where A. baumannii has become a serious health problem, particularly in the intensive care unit, where it can cause serious and prolonged outbreaks (Gonzalez-Villoria and ValverdeGarduno, 2016). A. baumannii is often multidrug resistant (Peleg et al., 2008; Gonzalez-Villoria and Valverde-Garduno, 2016) making antimicrobial therapy of A. baumannii infections difficult. 
In some cases, with the advent of resistance to last line antibiotics such as colistin, there are few therapeutic options left (Higgins et al., 2010; Manchanda et al., 2010; Göttig et al., 2014; Cayô et al., 2016).

Acinetobacter baumannii is known to have a great genome plasticity, which is the capacity to acquire and disseminate genes, especially those related to antimicrobial resistance which are commonly associated with insertion sequence (IS) elements in transposons and plasmids; this dynamism in the genome of A. baumannii contributed to the rapid evolution of drug resistance (Adams et al., 2010) as has been demonstrated for ISAba1 mobilizing antimicrobial resistance genes (Mugnier et al., 2009). These processes are achieved thanks to mobile genetic elements (MGEs) harboring resistance genes. The simplest MGEs are ISs, that can also form transposons (Tn), and there are more complex structures such as integrons, resistance islands (RI), and plasmids. Antimicrobial resistance genes are often integrated into resistance cassettes related to translocation elements, causing cumulative resistance to multiple drugs (Roca et al., 2012).

A diverse range of MGEs have been described in A. baumannii, for example transposons such as Tn2008, Tn2008B, Tn2006, Tn2009, or Tn2007, which represent different transposon configurations carrying the $b l a_{\mathrm{OXA}}-23$ gene together with ISAba1 or ISAba4, and additional genes (Nigro and Hall, 2016). Great variability in antimicrobial resistance platforms, including MGEs, have been recorded even within the same international clone (IC), illustrating their contribution to the evolution of drug resistance (Adams et al., 2010). Plasmids in Acinetobacter spp. are unique and unrelated to those from other genera, although they often share the same resistance determinants, such as $\operatorname{str} A, \operatorname{str} B, \operatorname{tet}(B)$ or sul2. In $A$. baumannii, a diverse array of plasmids have been found, ranging in size from $2 \mathrm{~Kb}$ to more than $150 \mathrm{~Kb}$. The larger plasmids normally encode for more than one resistance gene, but up to now little is known about these plasmids (Carattoli, 2013; Hamidian et al., 2016).

The aim of this study was to characterize the MGEs such as plasmids and RI of three different A. baumannii clinical isolates, representing different clonal lineages.

\section{MATERIALS AND METHODS}

\section{Bacterial Isolates}

Three A. baumannii isolates recovered from two hospitals in Cochabamba, Bolivia, in September 2015, January 2016, and October 2016 (Table 1) representing three different ICs (IC4, IC5. and IC7) were selected for this study. We previously reported their carbapenem resistance mechanisms and molecular epidemiology (Cerezales et al., 2019).

\section{Antimicrobial Susceptibility Testing}

In addition to previously reported carbapenem susceptibility testing results, in the present study we investigated the following antimicrobials by agar dilution: amikacin, azithromycin, chloramphenicol, trimethoprim-sulfamethoxazole, erythromycin, levofloxacin, minocycline, kanamycin, and tetracycline.

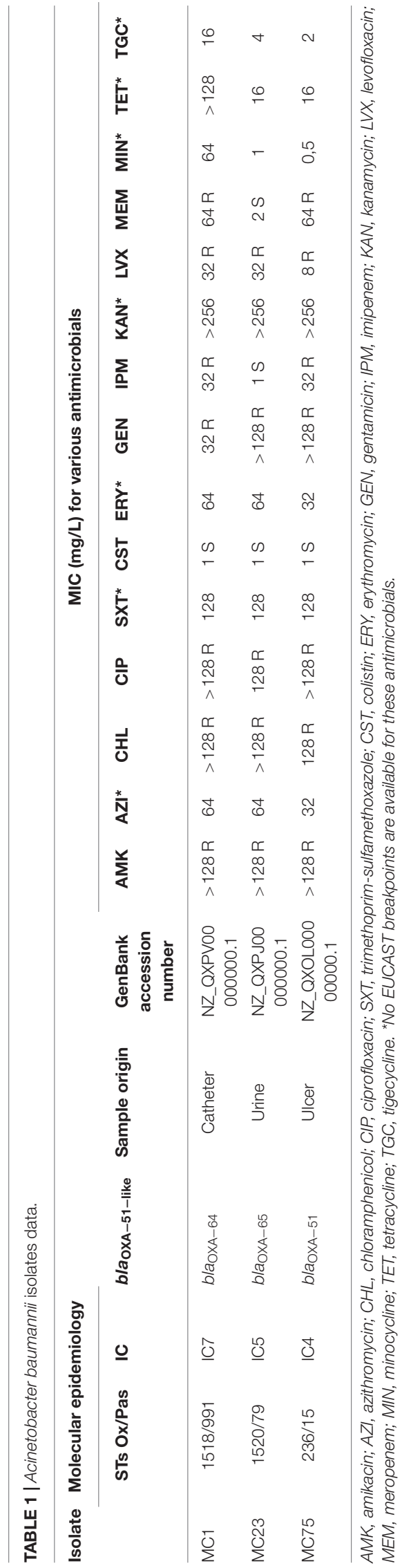


TABLE 2 | Plasmid content, size, location resistance genes as determined by WGS, and accession numbers.

\begin{tabular}{|c|c|c|c|c|c|}
\hline \multirow[b]{2}{*}{ Isolate } & \multirow[b]{2}{*}{ Plasmids } & \multirow[b]{2}{*}{$\begin{array}{c}\text { Accession } \\
\text { number }\end{array}$} & \multirow[b]{2}{*}{ Size } & \multicolumn{2}{|c|}{ Location of resistance genes } \\
\hline & & & & Plasmid & Chromosome \\
\hline \multirow[t]{2}{*}{ MC1 } & pMC1.1 & MK531536 & $184 \mathrm{~Kb}$ & $\begin{array}{c}\text { strA } \\
\text { strB } \\
\text { aac(3)-Ila } \\
\operatorname{aac}\left(6^{\prime}\right)-l a n \\
\operatorname{tet}(B)\end{array}$ & blaOXA-23 \\
\hline & pMC1.2 & MK531537 & $8.7 \mathrm{~Kb}$ & sul2 & \\
\hline \multirow[t]{2}{*}{ MC23 } & $\begin{array}{l}\text { pMC23.1 } \\
\text { pMC23.2 }\end{array}$ & $\begin{array}{l}\text { MK531538 } \\
\text { MK531537 }\end{array}$ & $\begin{array}{l}67 \mathrm{~Kb} \\
8.7 \mathrm{~Kb}\end{array}$ & & $\begin{array}{l}\text { strA } \\
\text { strB }\end{array}$ \\
\hline & pMC23.3 & MK531539 & $6 \mathrm{~Kb}$ & $\operatorname{aadB}$ & $\begin{array}{l}\text { sul2 } \\
\text { floR } \\
\text { aadA1 } \\
\text { sat2 } \\
\text { dfrA1 }\end{array}$ \\
\hline MC75 & pMC75.2 & MK531541 & $13.9 \mathrm{~Kb}$ & $\begin{array}{l}\text { bla TEM-1 } \\
\text { aac(3)-Ila }\end{array}$ & \\
\hline
\end{tabular}

FIGURE 1 | Resistance island Rl1.MC23 in isolate MC23. Arrows represent predicted ORFs and the direction of the arrow represents the direction of transcription.
Resistance genes are shown by orange arrows and transposon-related genes, recombinases, and insertion sequences are indicated by green arrows. Genes
involved in plasmid mobility are shown in pink. Other genes are indicated by gray arrows. Hypothetical proteins are not shown.

FIGURE 2 | Resistance island RI2.MC23 in isolate MC23. Arrows represent predicted ORFs and the direction of the arrow represents the direction of transcription.
Resistance genes are shown by orange arrows and transposon-related genes, recombinases, and insertion sequences are indicated by green arrows. Other genes
are indicated by gray arrows. Hypothetical proteins are not shown.

MICs were interpreted using the European Committee on Antimicrobial Susceptibility Testing (EUCAST) breakpoints ${ }^{1}$.

\section{MinION Long-Read Sequencing and Assembly}

The Oxford Nanopore Technologies (Oxford, United Kingdom) MinION sequencer was used to obtain long reads to span repetitive elements and close genomes and plasmids. DNA extraction was performed using the Genomic-tip 100/G kit

${ }^{1}$ http://www.eucast.org/clinical_breakpoints/
(Qiagen, Hilden, Germany). Library preparation was carried out according to manufacturer's indications using a combination of Native Barcoding Kit 1D and Ligation Sequencing Kit 1D; EXPNBD103 and SQK-LSK108 (Oxford Nanopore Technologies, Oxford, United Kingdom), respectively.

The tool Albacore (Oxford Nanopore Technologies, Oxford, United Kingdom) was used for demultiplexing the reads which were later used to perform the Canu assembly (Koren et al., 2017). A hybrid assembly combining previous MiSeq short reads with MinION-generated long reads was performed using a hybridSpades (Antipov et al., 2016). 


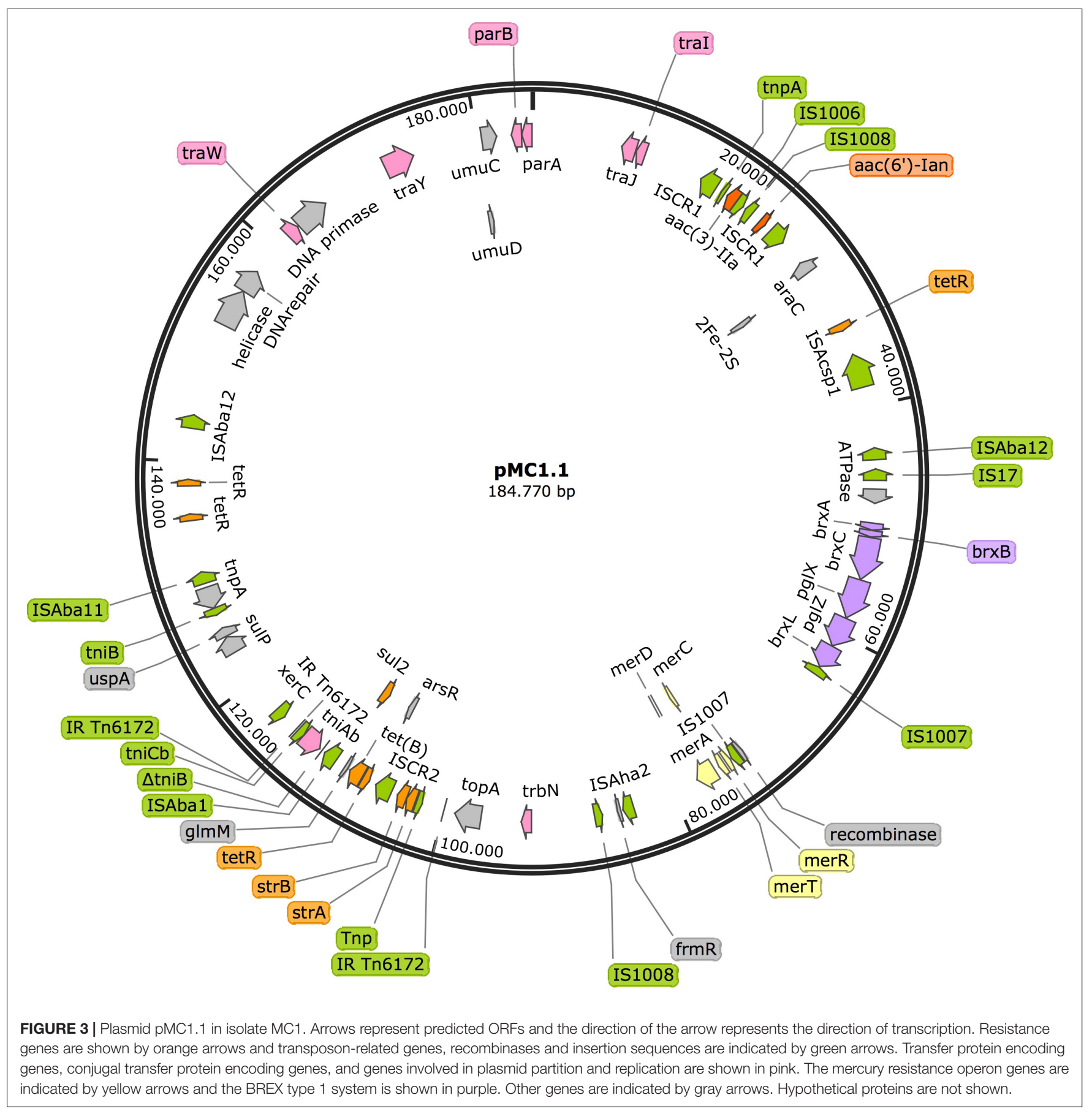

\section{Plasmid Annotation and Visualization}

ORFfinder $(\mathrm{NCBI})^{2}$ was used to predict the open reading frames (ORF) of the plasmids. A second functional annotation of the genomes was performed using the online tool Rapid Annotation Subsystem Technology (RAST) ${ }^{3}$ (Genomics et al., 2008). Subsequently, the tool SnapGene Viewer (GSL Biotech) ${ }^{4}$

\footnotetext{
${ }^{2}$ https://www.ncbi.nlm.nih.gov/orffinder/

${ }^{3}$ https://rast.nmpdr.org/

${ }^{4} \mathrm{https}: / /$ www.snapgene.com
}

was used to obtain a circular diagram of the plasmids. Graphic comparisons between similar plasmids, pMC1.1 and pA297-3, as well as pMC23.1 and pAC30c, were carried out with the tool Kablammo (Wintersinger and Wasmuth, 2015).

\section{Conjugation Experiments}

Broth mate conjugation experiments were performed to determine the location of antimicrobial resistance genes using the sodium azide-resistant Escherichia coli J53 and the rifampicin-resistant A. baumannii BM4547 as recipient 


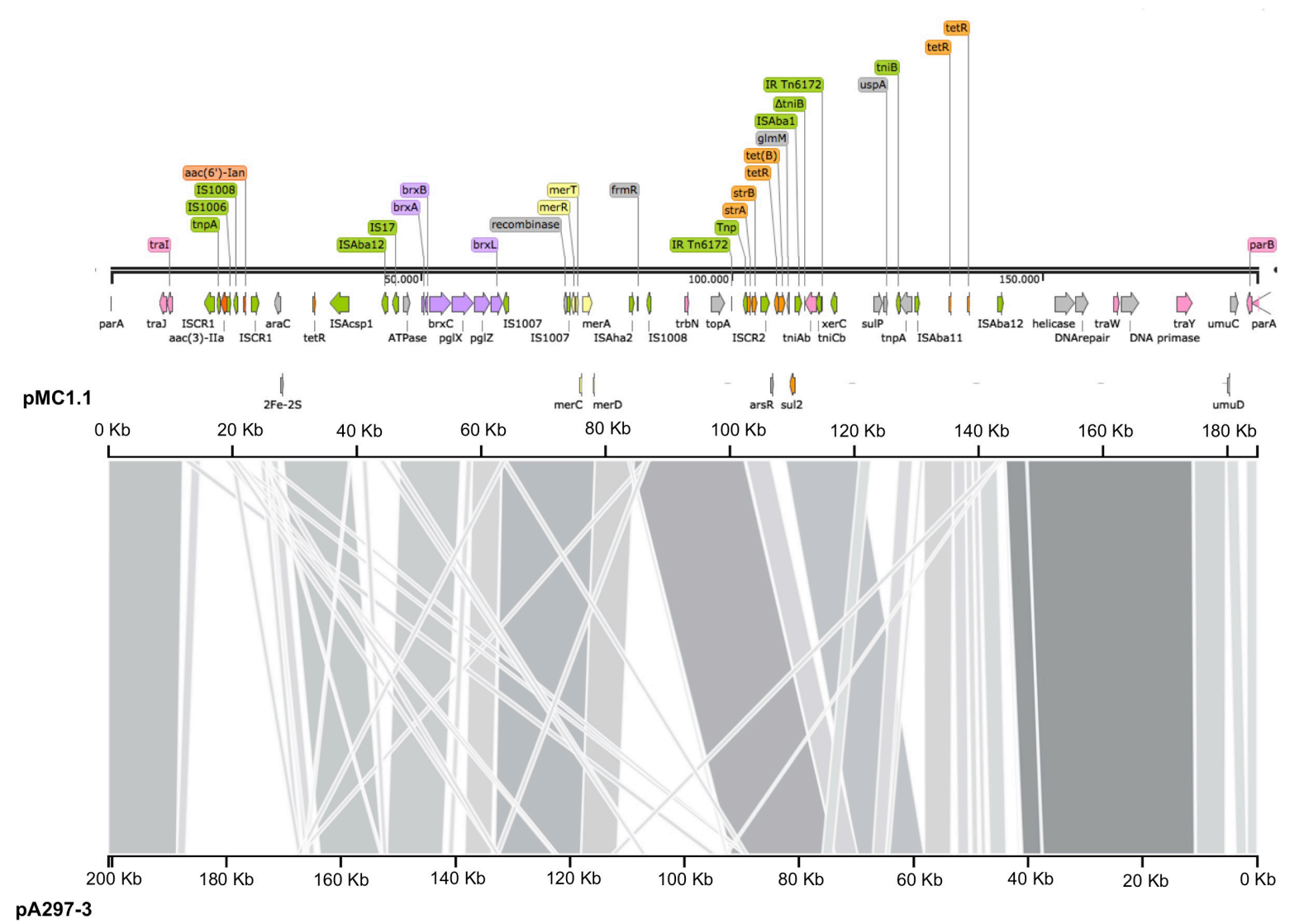

FIGURE 4 | Comparison of plasmids pMC1.1 and pA297-3. The top axis represents the pMC1.1, the bottom axis represents pA297-3. Gray shaded regions show the homologous regions between the two plasmids.

strains. Selection of E. coli $\mathrm{J} 53$ transconjugants was performed using sodium azide $(200 \mathrm{mg} / \mathrm{L})$ combined either with amikacin $(30 \mathrm{mg} / \mathrm{L})$, streptomycin $(30 \mathrm{mg} / \mathrm{L})$, kanamycin $(30 \mathrm{mg} / \mathrm{L})$, gentamicin $(30 \mathrm{mg} / \mathrm{L})$, or ticarcillin $(100 \mathrm{mg} / \mathrm{L})$, and selection for A. baumannii BM4547 was performed using rifampicin $(60 \mathrm{mg} / \mathrm{L})$ combined with gentamicin $(30 \mathrm{mg} / \mathrm{L})$ or ticarcillin $(100 \mathrm{mg} / \mathrm{L}$. Transconjugants were selected with the antimicrobials to select for the plasmids encoding their respective resistance genes. Strain MC1 was resistant to rifampicin, therefore conjugation with A. baumannii BM4547 could not be performed. The transconjugants were tested by PCR for the bla TEM gene.

\section{RESULTS AND DISCUSSION}

MC1 and MC75 were previously tested as carbapenem-resistant and carried the carbapenemase encoding $b l a_{\mathrm{OXA}-23-\text { like }}$ gene (Cerezales et al., 2019). Further testing revealed that MC1 and MC75 were also resistant to amikacin, chloramphenicol, ciprofloxacin, gentamicin, and levofloxacin. MC23 was resistant to amikacin, chloramphenicol, ciprofloxacin, gentamicin, and levofloxacin but was susceptible to carbapenems. All three isolates were susceptible to colistin (Table 1).
The bla $a_{\mathrm{OXA}-23}$ encoding gene was located on the chromosome in a Tn2008 vehicle in the isolates MC1 and MC75 (Table 2).

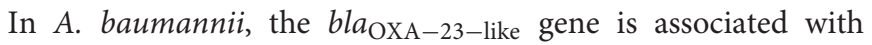
ISAba1, which contributes to its overexpression as well as its mobilization (Nigro and Hall, 2016). Tn2008 has previously been described in Bolivian A. baumannii isolates and this mirrors the spread of this structure among different ICs leading to a carbapenem-resistant phenotype (Nigro and Hall, 2016; Sennati et al., 2016; Chen et al., 2017; Ewers et al., 2017; Cerezales et al., 2018).

\section{Resistance Islands}

In the isolate MC23, the gene strA was located on a resistance island in the chromosome (RI1.MC23) (accession number MK531542), together with other antimicrobial resistance genes such as sul2, floR, and $s t r B$. Diverse IS elements were found, with the resistance island bracketed by two copies of a transposase from the IS4 family in reverse orientation (Figure 1). Two genes involved in conjugation were also present in this structure, suggesting a plasmid origin.

In addition, a second chromosomal resistance island was also found in this isolate (RI2.MC23) (accession number MK531543), that carried a typical structure from class 2 integrons, 


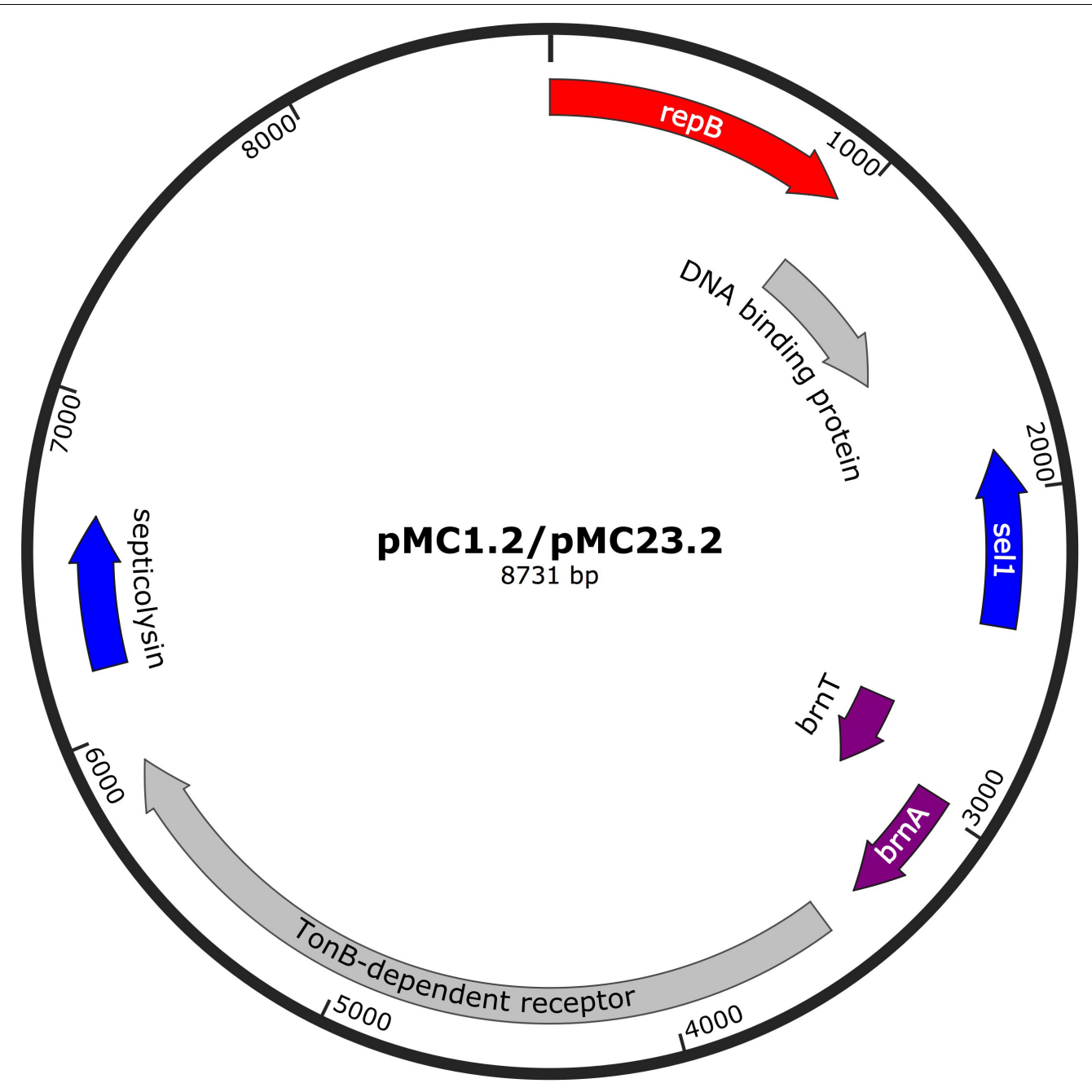

FIGURE 5 | Plasmids pMC1.2 and pMC23.2 in isolates MC1 and MC23, respectively. Arrows represent predicted ORFs and the direction of the arrow represents the direction of transcription. Red arrow is used for the replicon. The toxin-antitoxin system is indicated by violet arrows and blue arrows represent virulence genes. Other genes are shown in gray.

$d f r A$-sat2-aadA1-ybeA-ybfAybfB-ybgA, located between the $\operatorname{Tn} 7$ transposition module tnsABCDE and a non-functional Intl2 integrase. Additionally, a Tn3 transposon was found inserted in the Tn7 transposon, carrying three genes, $\operatorname{tn} p A$, encoding for a Tn3 transposase; $\operatorname{tn} p R$ encoding a Tn3 resolvase; and the antimicrobial resistance gene bla $a_{\mathrm{TEM}-1 \mathrm{~A}}$ (Figure 2).

The gene encoding Apha6 was found on the chromosome of MC75 bracketed by two ISAba125 that is a composite transposon known as TnaphA6 (Matos et al., 2019).

\section{Plasmids pMC1.1}

Annotation of pMC1.1 (accession number MK531536), 39\% GC content, revealed many different IS elements such as IS1006, IS1007, IS1008, ISAcsp1, IS91 family, ISAha2, ISAba11, ISAba12, and IS17. This plasmid carried a mercuric resistance operon, similar to an already described mercuric $\mathrm{Tn}$ in a $200 \mathrm{~Kb}$ plasmid (pA297-3) from an IC1 A. baumannii isolate, but it lacks the merP open reading frame (Hamidian et al., 2016). Different antimicrobial resistance determinants such as $\operatorname{str} A, \operatorname{str} B, \operatorname{aac}(3)-I I a$, and $a a c\left(6^{\prime}\right)$-Ian, conferring resistance to aminoglycosides, sul2 conferring resistance to sulphonamides, and tet $(B)$ conferring resistance to tetracycline were also present. The region of the plasmid carrying $\operatorname{str} A$, strB, and sul2 shared high homology with Tn6172, located in pA297-3 as well (Figure 3), however, in pMC1.1 arsR, tet $R$, and tet(B) genes were also located within Tn6172 with an ISCR2 transposable element (IS91 family). This ISCR element has been described associated with different antimicrobial resistance genes in A. baumannii, especially with sul2, contributing to their mobilization thanks to a rolling circle transposition mechanism (Toleman et al., 2006), and was similar to other plasmids from Argentina (Vilacoba et al., 2013) and to plasmids found in an ST25 isolate from Australia (Hamidian and Hall, 2016). However, the location of tetR-tet $B$ genes was different; they were located between $\operatorname{glm} M$ and $\operatorname{ars} R$, suggesting a possible later insertion of these genes in 


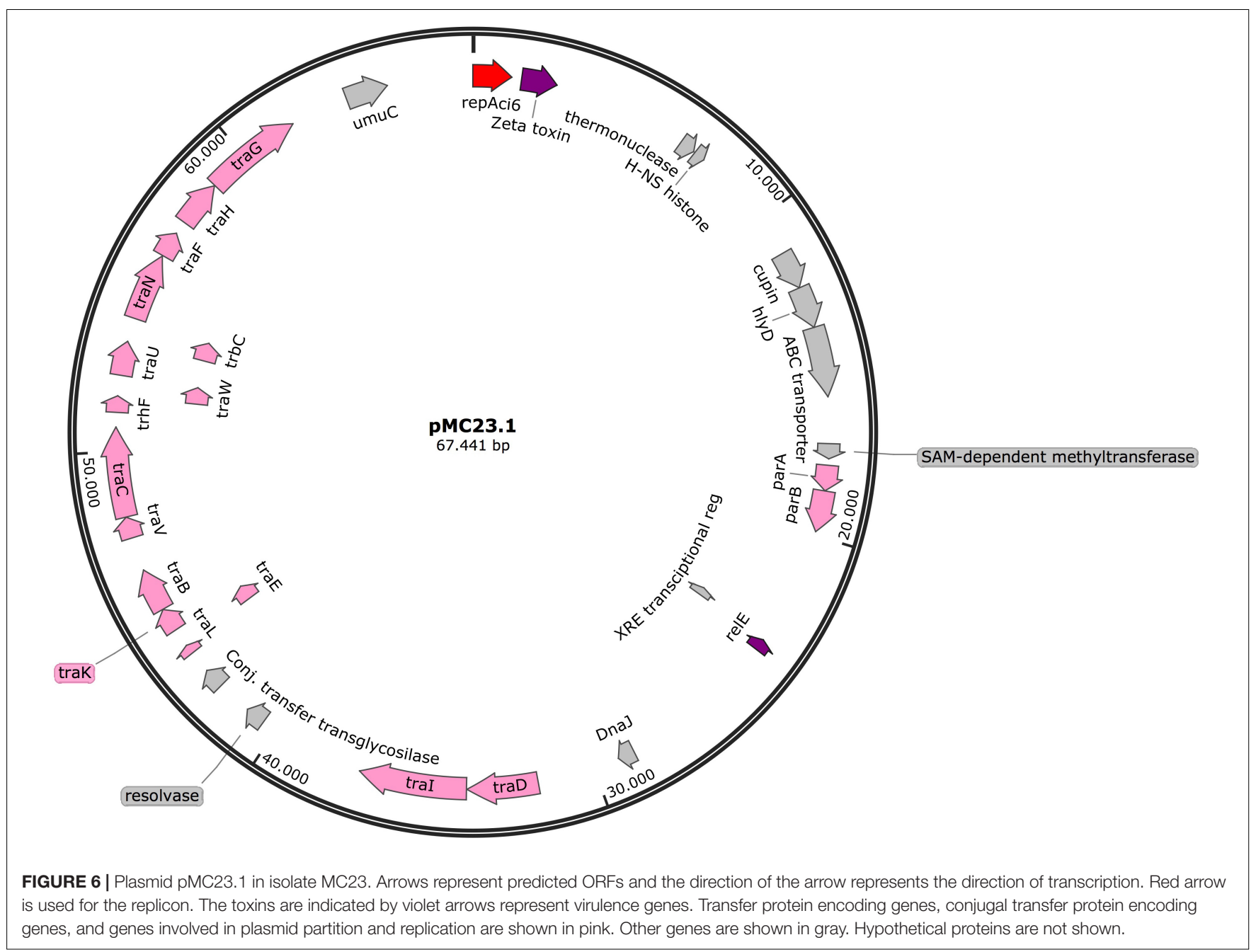

different positions within the transposon (Vilacoba et al., 2013). In addition, the same inverted repeats (IR) generated by the insertion of the transposon were also found in pMC1.1 which together with the similar backbone with pA297-3 (Figure 4) suggest they share a common origin. The genes aac(3)-IIa and $\operatorname{aac}\left(6^{\prime}\right)$-Ian were associated with IS6 family IS and bracketed by two ISCR 1 in inverted orientation. ISCR 1 belongs to the IS91 family and has been described related to class 1 integrons and antimicrobial resistance genes in diverse Gram-negative species such as Klebsiella pneumoniae, P. aeruginosa, and Citrobacter freundii (Toleman et al., 2006). Different transfer genes (tra) were also found in this plasmid, as well as genes involved in plasmid partition and replication ( $\operatorname{par} B / \operatorname{rep} B$ and $x e r C$ ) that are related to segregational stability of plasmids. This plasmid also encoded a system called BREX type 1 (bacteriophage exclusion) which has been described to be involved in phage resistance (Goldfarb et al., 2015).

\section{pMC1.2/pMC23.2}

The $8.7 \mathrm{~Kb}$ plasmids found in MC1 and MC23 (pMC1.2 and pMC23.2) were identical (accession number MK531537), with a GC content of $34.3 \%$ (Figure 5). This small plasmid has often been found in IC1 A. baumannii isolates (Lean and Yeo, 2017). Annotation of this plasmid revealed ORFs encoding for a RepB replicon (Rep-3 superfamily, GR2) (Bertini et al., 2010; Lean and Yeo, 2017) a toxin-antitoxin system (BrnT-BrnA), that is involved in vertical stability; TonB-dependent receptor, related to the transmission of signals from the outside of the cell leading to transcriptional activation of target genes; a septicolysin gene encoding a cytolytic enzyme toward eukaryotic cells and is involved in pathogenesis; as well as sel1 gene that encodes for a protein that has been described in diverse prokaryotic genera and has an important role in virulence.

\section{pMC23.1}

The largest plasmid in MC23 was the $67.5 \mathrm{~Kb}$ pMC23.1 (accession number MK531538) (Figure 6). It belonged to GR6 according to its replicase, repAci6. Its GC content was 33.7\% and almost all of its putative protein encoding genes were related to conjugative plasmid transfer in a tra locus, some of them are part of a type IV (T4SS) secretion system. This T4SS is able to secrete or take up both proteins and DNA, and possibly is involved in natural competence, a feature of A. baumannii (Salto et al., 2018). Two toxin encoding genes were present in the plasmid, 


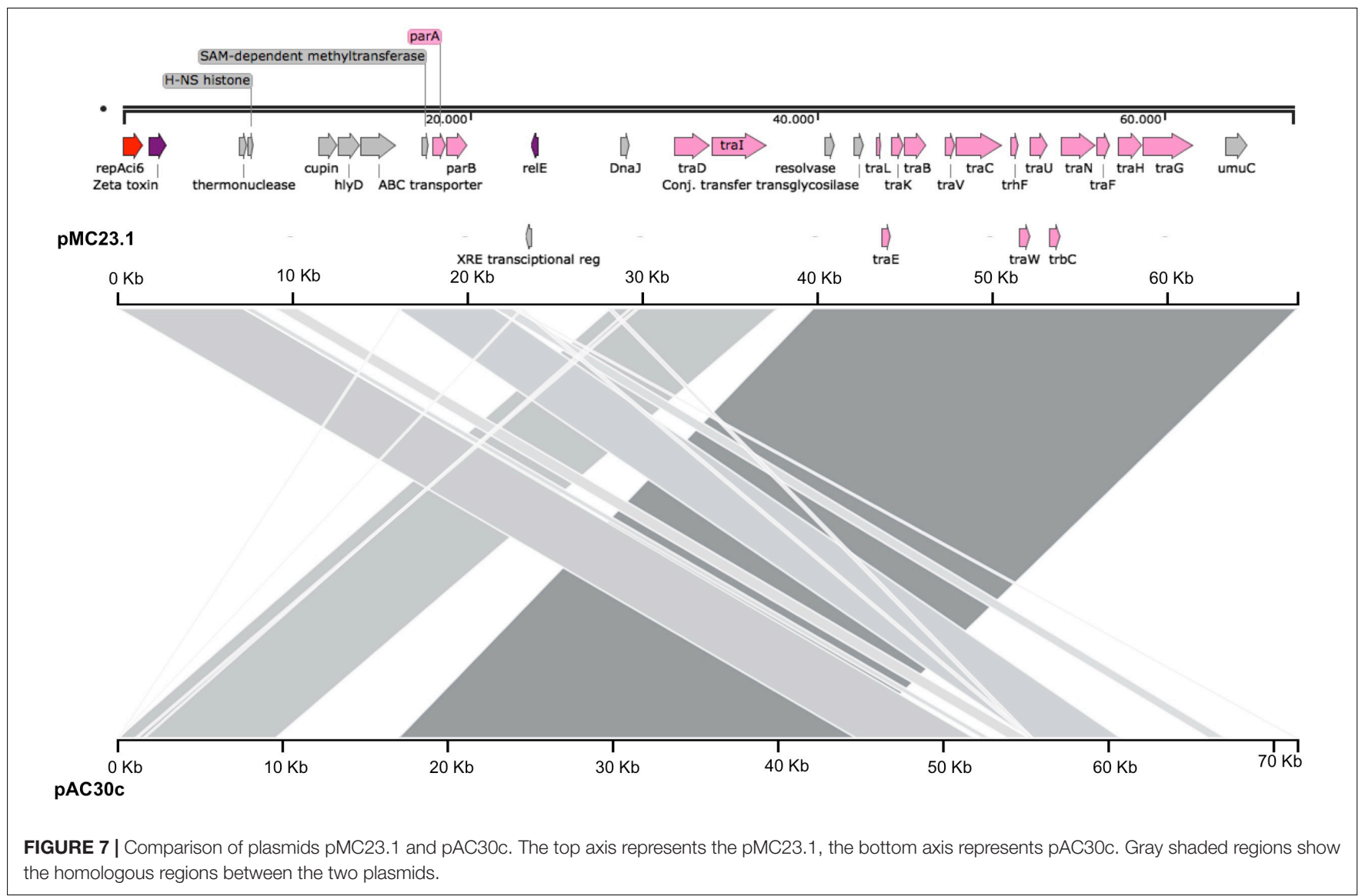

relE and zeta toxin, but no antitoxins were found, although they were present in a very similar plasmid (pAC30c) in an A. baumannii isolate belonging to ST195 (IC2) (Figure 7; Lean et al., 2016). In addition, the partition genes $\operatorname{par} A / p a r B$ were also encoded on pMC23.1. The backbone of pMC23.1 and pAC30c were very similar, with only a few differences. pMC23.1 lacked some hypothetical proteins present in $\mathrm{pAC} 30 \mathrm{c}$, and the region encoding for tellurite resistance (telA gene and IS66); while traD, a cupin-like protein (that is a superfamily of enzymes including dioxygenases, decarboxylases, hydrolases, or isomerases); HlyD protein, that exports proteins from the cytosol to the outside of the cell, and an $\mathrm{ABC}$ transporter were not present in pAC30.

\section{pMC23.3}

A $6 \mathrm{~Kb}$ small plasmid was present in the isolate MC23, pMC23.3 (accession number MK531539), 39.2\% GC content, and was found to have $100 \%$ similarity with an already described plasmid, pRAY from an isolate in South Africa, encoding resistance to gentamicin, kanamycin and tobramycin ( $a a d B$ gene) together with mobA and mobC genes, which are thought to encode mobilization proteins (Lean and Yeo, 2017). Many similar plasmids have been found in diverse A. baumannii isolates from different ICs and countries, suggesting a common origin and subsequent diversification in their evolution. Concurrent with other studies, no rep gene was found in the plasmid sequence, supporting the idea of the presence of a mechanism of replication relying in the host RNA polymerase (Lean and Yeo, 2017).

\section{pMC75.1}

Analysis of pMC75.1 (accession number MK531540) a large plasmid of $150 \mathrm{~Kb}$ revealed that it was very similar to pMC1.1 (sharing $80 \%$ of their sequences), it also carried a Tn6172, in which antimicrobial resistance genes such as $s u l 2, \operatorname{str} B$, and $\operatorname{str} A$ are encoded, but lacking tet(B) and arsR that were present in pMC1.1 (Figure 8). The mer operon was also found in this plasmid, and many genes encoding conjugative transfer proteins. The BREX type 1 system was also present. A stbA gene was found, the protein encoded by this gene plays a role in plasmid stability as well as $p a r A / p a r B$. Several IS elements were also present, i.e., ISAba1, ISAba125, ISAba14, ISAba42, IS1007, and ISAha2. However, this plasmid lacked the transposon carrying aac(3)-IIa and $\operatorname{aac}\left(6^{\prime}\right)$-Ian.

\section{pMC75.2}

The 13.9 Kb plasmid, pMC75.2 (accession number MK531541) (Figure 9) with a GC content of $40.3 \%$, carried the broadspectrum $\beta$-lactamase $b l a_{\mathrm{TEM}-1 \mathrm{~B}}$ and the aminoglycoside resistance gene aac(3)-IIa flanked on both sides by IS15DIV; a toxin-antitoxin system, brnT/brnA; a TonB-dependant receptor, a septicolysin gene and $m o b A / m o b S$, which are involved in plasmid mobility. Conjugation experiments revealed that 


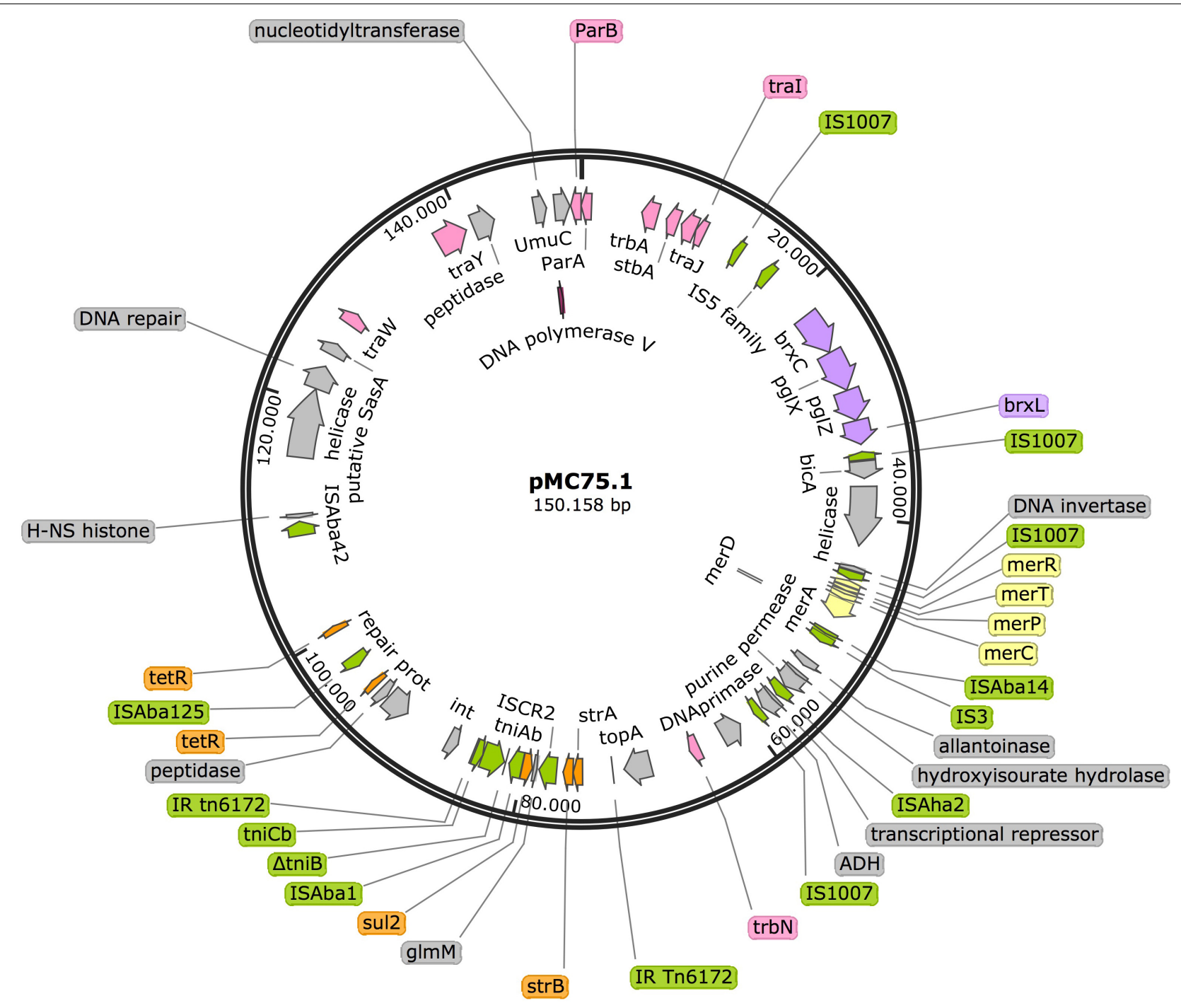

FIGURE 8 | Plasmid pMC75.1 in isolate MC75. Arrows represent predicted ORFs and the direction of the arrow represents the direction of transcription. Resistance genes are shown by orange arrows and transposon-related genes, recombinases and insertion sequences are indicated by green arrows. Transfer protein encoding genes, conjugal transfer protein encoding genes, and genes involved in plasmid partition and replication are shown in pink. The mercury resistance operon genes are indicated by yellow arrows and the BREX type 1 system is shown in purple. Other genes are indicated by gray arrows. Hypothetical proteins are not shown.

pMC75.2 was transferable into A. baumannii BM4547 but it was unstable and was lost after several passages. The replicon of this plasmid belonged to the RepB (Rep_3) superfamily with $100 \%$ homology. This plasmid shares a great homology with pMC1.2/pMC23.2, same RepB, toxin-antitoxin system, TonB-dependant receptor and septicolysin; it seems that one of them has lost or alternatively acquired the integron carrying the antimicrobial resistance genes and the mobility genes.

Recently, two similar plasmids to pMC75.1 and pMC75.2 were described in a Brazilian A. baumannii isolate representing the same ST (ST15). This illustrates that these plasmids can be very plastic by acquiring or losing genes, but can also be conserved within a ST (Matos et al., 2019).

The two carbapenem-resistant isolates carried the bla $a_{\text {OXA-23 }}$ gene in Tn2008, which has been previously described in diverse ICs (Nigro and Hall, 2016; Ewers et al., 2017) including IC7 isolates recovered from a hospital in the same city, Cochabamba (Sennati et al., 2016). The Tn2008 contributes to the overexpression of the carbapenemase encoding gene and to its mobilization. In addition, all three isolates harbored three aminoglycoside resistance genes such as $\operatorname{aac}(3)-I I a, \operatorname{str} A$, and $s \operatorname{tr} B$; and sul2 conferring resistance to sulphonamides; MC1 carried tet $B$ conferring resistance to tetracycline as well. All the genes were found to be associated with IS elements, constituting transposons that lead to their mobilization and make genetic rearrangements more likely to happen. These genes were found both in the chromosome and in plasmids, demonstrating the plasticity of the A. baumannii genome and the mobility of these antimicrobial resistance 


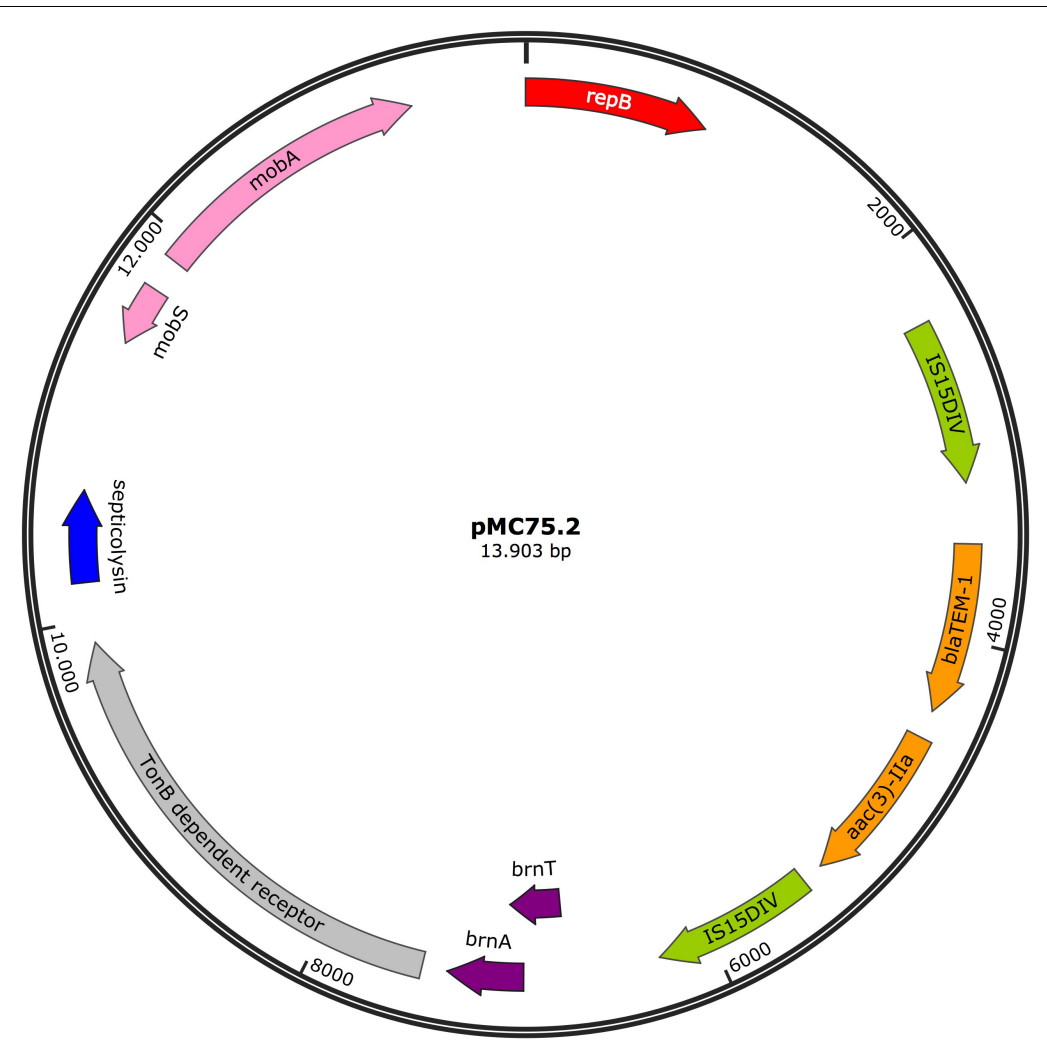

FIGURE 9 | Plasmid pMC75.2 in isolate MC75. Arrows represent predicted ORFs and the direction of the arrow represents the direction of transcription. Resistance genes are shown by orange arrows and insertion sequences are indicated by green arrows. Genes involved in plasmid mobility are shown in pink. The toxin-antitoxin system is shown in violet. Blue represents virulence genes. Other genes are indicated by gray arrows. Hypothetical proteins are not shown. Red arrow is used for the replicon.

determinants within MGEs such as transposons or plasmids.

\section{CONCLUSION}

In summary, these data further confirm that A. baumannii has a great ability to acquire antimicrobial resistance determinants and become a threat in hospitals. These are associated with different plasmids and many different IS elements, of which some are found in multiple genera. For these reasons it is important to study the dynamics and resistomes of the bacterial populations in order to understand the situation in each hospital or unit. The fact that some of these plasmids have been found in diverse A. baumannii clonal lineages mirrors the transfer and prevalence of these MGEs contributing to the spread of antimicrobial resistance worldwide.

\section{DATA AVAILABILITY STATEMENT}

The datasets generated for this study can be found in the GenBank, MK531536, MK531538, MK531537, MK531539, MK531540, and MK531541.

\section{AUTHOR CONTRIBUTIONS}

MC, KX, JW, and $\mathrm{PH}$ contributed to the design of the experiments. MC, KX, and JW performed the experiments. $\mathrm{MC}, \mathrm{KX}, \mathrm{JW}, \mathrm{OK}, \mathrm{HS}, \mathrm{LG}$, and $\mathrm{PH}$ analyzed and interpreted the data. $\mathrm{MC}, \mathrm{KX}$, and $\mathrm{PH}$ wrote the manuscript. All authors contributed to critical manuscript revision, read, and approved the submitted version.

\section{FUNDING}

This work was supported by the Basque Government 1129 and University of the Basque Country [Grupo Consolidado del Sistema Universitario Vasco (IT1097-16)/UPV/EHU GIC15/143]. PH was supported by the German Research Council (DFG) - FOR2251 (www.acinetobacter.de). This work was supported by the German Center for Infection Research (DZIF).

\section{ACKNOWLEDGMENTS}

We would like to thank Yvonne Pfeifer for providing the conjugation protocol and also Rémy A. Bonnin for providing the A. baumannii BM4547 strain. 


\section{REFERENCES}

Adams, M. D., Chan, E. R., Molyneaux, N. D., and Bonomo, R. A. (2010). Genomewide analysis of divergence of antibiotic resistance determinants in closely related isolates of Acinetobacter baumannii. Antimicrob. Agents Chemother. 54, 3569-3577. doi: 10.1128/AAC.00057-10

Antipov, D., Korobeynikov, A., Mclean, J. S., and Pevzner, P. A. (2016). Genome analysis HYBRIDSPADES: an algorithm for hybrid assembly of short and long reads. Bioinformatics 32, 1009-1015. doi: 10.1093/bioinformatics/btv688

Bertini, A., Poirel, L., Mugnier, P. D., Villa, L., Nordmann, P., and Carattoli, A. (2010). Characterization and PCR-based replicon typing of resistance plasmids in Acinetobacter baumannii. Antimicrob. Agents Chemother. 54, 4168-4177. doi: 10.1128/AAC.00542-10

Carattoli, A. (2013). Plasmids and the spread of resistance. Int. J. Med. Microbiol. 303, 298-304. doi: 10.1016/j.ijmm.2013.02.001

Cayô, R., Rodrigues-Costa, F., Pereira Matos, A., Godoy Carvalhaes, C., Dijkshoorn, L., and Gales, A. C. (2016). Old clinical isolates of Acinetobacter seifertii in Brazil producing OXA-58. Antimicrob. Agents Chemother. 60, 25892591. doi: 10.1128/AAC.01957-15

Cerezales, M., Ocampo-Sosa, A. A., Álvarez Montes, L., Díaz Ríos, C., Bustamante, Z., Santos, J., et al. (2018). High prevalence of extensively drug-resistant Acinetobacter baumannii at a children hospital in Bolivia. Pediatr. Infect. Dis. J. 37, 1118-1123. doi: 10.1097/INF.0000000000001962

Cerezales, M., Xanthopoulou, K., Wille, J., Bustamante, Z., Seifert, H., Gallego, L., et al. (2019). Acinetobacter baumannii analysis by core genome MLST in two hospitals in Bolivia: endemicity of international clone 7 isolates (CC25). Int. J. Antimicrob. Agents 53, 844-849. doi: 10.1016/j.ijantimicag.2019.03.019

Chen, Y., Gao, J., Zhang, H., and Ying, C. (2017). Spread of the blaOXA-23containing Tn2008 in carbapenem-resistant Acinetobacter baumannii isolates grouped in CC92 from China. Front. Microbiol. 8:163. doi: 10.3389/fmicb.2017. 00163

Ewers, C., Klotz, P., Leidner, U., Stamm, I., Prenger-Berninghoff, E., Göttig, S., et al. (2017). OXA-23 and ISAba1 -OXA-66 class D $\beta$-lactamases in Acinetobacter baumannii isolates from companion animals. Int. J. Antimicrob. Agents 49, 37-44. doi: 10.1016/j.ijantimicag.2016.09.033

Genomics, B., Aziz, R. K., Bartels, D., Best, A. A., DeJongh, M., Disz, T., et al. (2008). The RAST server: rapid annotations using subsystems technology. BMC Genomics 9:75 doi: 10.1186/1471-2164-9-75

Goldfarb, T., Sberro, H., Weinstock, E., Cohen, O., Doron, S., Charpak-Amikam, Y., et al. (2015). BREX is a novel phage resistance system widespread in microbial genomes. EMBO J. 34, 169-183. doi: 10.15252/embj.201489455

Gonzalez-Villoria, A. M., and Valverde-Garduno, V. (2016). Antibiotic-resistant Acinetobacter baumannii increasing success remains a challenge as a nosocomial pathogen. J. Pathog. 2016:7318075. doi: 10.1155/2016/7318075

Göttig, S., Gruber, T. M., Higgins, P. G., Wachsmuth, M., Seifert, H., and Kempf, V. A. J. (2014). Detection of pan drug-resistant Acinetobacter baumannii in Germany. J. Antimicrob. Chemother. 69, 2578-2579. doi: 10.1093/jac/dku170

Hamidian, M., Ambrose, S. J., and Hall, R. M. (2016). A large conjugative Acinetobacter baumannii plasmid carrying the sul2 sulphonamide and $\operatorname{str} A B$ streptomycin resistance genes. Plasmid 87-88, 43-50. doi: 10.1016/j.plasmid. 2016.09.001

Hamidian, M., and Hall, R. M. (2016). The resistance gene complement of D4, a multiply antibiotic-resistant ST25 Acinetobacter baumannii isolate, resides in two genomic islands and a plasmid. J. Antimicrob. Chemother. 71, 1730-1741. doi: $10.1093 / \mathrm{jac} / \mathrm{dkw041}$

Higgins, P. G., Dammhayn, C., Hackel, M., and Seifert, H. (2010). Global spread of carbapenem-resistant Acinetobacter baumannii. J. Antimicrob. Chemother. 65, 233-238. doi: 10.1093/jac/dkp428
Koren, S., Walenz, B. P., Berlin, K., Miller, J. R., Bergman, N. H., and Phillippy, A. M. (2017). Canu: scalable and accurate long-read assembly via adaptive $k$ mer weighting and repeat separation. Genome Res. 27, 722-736. doi: 10.1101/ gr.215087.116

Lean, S. S., and Yeo, C. C. (2017). Small, enigmatic plasmids of the nosocomial pathogen, Acinetobacter baumannii: good, bad, who knows? Front. Microbiol. 8:1547. doi: 10.3389/fmicb.2017.01547

Lean, S.-S., Yeo, C. C., Suhaili, Z., and Thong, K.-L. (2016). Comparative genomics of two ST 195 carbapenem-resistant Acinetobacter baumannii with different susceptibility to polymyxin revealed underlying resistance mechanism. Front. Microbiol. 6:1445. doi: 10.3389/fmicb.2015.01445

Manchanda, V., Sanchaita, S., and Singh, N. (2010). Multidrug resistant Acinetobacter. J. Glob. Infect. Dis. 2, 291-304. doi: 10.4103/0974-777X.68538

Matos, A. P., Cayô, R., Almeida, L. G. P., Streling, A. P., Nodari, C. S., Martins, W. M. B. S., et al. (2019). Genetic characterization of plasmid-borne bla OXA-58 in distinct Acinetobacter Species. mSphere 4:e00376-19. doi: 10.1128/mSphere. 00376-19

Mugnier, P. D., Poirel, L., and Nordmann, P. (2009). Functional analysis of insertion sequence ISAba1, responsible for genomic plasticity of Acinetobacter baumannii. J. Bacteriol. 191, 2414-2418. doi: 10.1128/JB.01258-08

Nigro, S. J., and Hall, R. M. (2016). Structure and context of Acinetobacter transposons carrying the oxa23 carbapenemase gene. J. Antimicrob. Chemother. 71, 1135-1147. doi: 10.1093/jac/dkv440

Peleg, A. Y., Seifert, H., and Paterson, D. L. (2008). Acinetobacter baumannii: emergence of a successful pathogen. Clin. Microbiol. Rev. 21, 538-582. doi: 10.1128/CMR.00058-07

Roca, I., Espinal, P., Vila-Farrés, X., and Vila, J. (2012). The Acinetobacter baumannii oxymoron: commensal hospital dweller turned pan-drug-resistant menace. Front. Microbiol. 3:148. doi: 10.3389/fmicb.2012.00148

Salto, I. P., Torres Tejerizo, G., Wibberg, D., Pühler, A., Schlüter, A., and Pistorio, M. (2018). Comparative genomic analysis of Acinetobacter spp. plasmids originating from clinical settings and environmental habitats. Science 8:7783. doi: 10.1038/s41598-018-26180-3

Sennati, S., Villagran, A. L., Bartoloni, A., Rossolini, G. M., and Pallecchi, L. (2016). OXA-23-producing ST25 Acinetobacter baumannii?: first report in Bolivia. J. Glob. Antimicrob. Resist. 4, 70-71. doi: 10.1016/j.jgar.2015.10.007

Toleman, M. A., Bennett, P. M., and Walsh, T. R. (2006). ISCR elements: novel gene-capturing systems of the 21st century? Microbiol. Mol. Biol. Rev. 70, 296-316. doi: 10.1128/MMBR.00048-05

Vilacoba, E., Almuzara, M., Gulone, L., Traglia, G. M., Figueroa, S. A., Sly, G., et al. (2013). Emergence and spread of plasmid-borne tet(B)::ISCR2 in minocyclineresistant Acinetobacter baumannii isolates. Antimicrob. Agents Chemother. 57, 651-654. doi: 10.1128/AAC.01751-12

Wintersinger, J. A., and Wasmuth, J. D. (2015). Kablammo: an interactive, webbased BLAST results visualizer. Bioinformatics 31, 1305-1306. doi: 10.1093/ bioinformatics/btu808

Conflict of Interest: The authors declare that the research was conducted in the absence of any commercial or financial relationships that could be construed as a potential conflict of interest.

Copyright (c) 2020 Cerezales, Xanthopoulou, Wille, Krut, Seifert, Gallego and Higgins. This is an open-access article distributed under the terms of the Creative Commons Attribution License (CC BY). The use, distribution or reproduction in other forums is permitted, provided the original author(s) and the copyright owner(s) are credited and that the original publication in this journal is cited, in accordance with accepted academic practice. No use, distribution or reproduction is permitted which does not comply with these terms. 